\title{
Electron beam-associated symmetric electrostatic solitary waves on the separatrix of magnetic reconnection: multi-spacecraft analysis
}

\author{
Shiyou Li, Shifeng Zhang, Hong Cai and Huabo Yang
}

\begin{abstract}
We present an in situ evidence of electron beam-associated symmetric bipolar electrostatic solitary waves (ESWs) on the current sheet-side of the separatrix of the magnetic reconnection in the near-Earth magnetotail by multi-spacecraft observation of Cluster. Within one spin period, 42 cases of symmetric ESWs are continuously observed during $2 \mathrm{~s}$ by SC2 while other spacecrafts do not "detect" them. And the Plasma Electron and Current Experiment (PEACE) spinPAD mode data exhibits unidirectional electron beam antiparallel to the ambient field, and no electron beam-like distribution is found by other spacecrafts without ESW observation. Though the electron beam is strongly associated with the ESWs in observation by multiple spacecraft differentiation, however, the relationship between the counter-directed electron beam and the simultaneously observed ESWs remains unclear and open to the next study.
\end{abstract}

Keywords: Magnetic reconnection; Electrostatic solitary wave; Electron beam

\section{Background}

The electrostatic solitary wave (ESW) has been regarded as one means of releasing the energy by carrying electrons out of the generating regions in the natural space. Magnetic reconnection (MR) presents an important mechanism in the space for the energy transformation from magnetic energy to kinetic energy and transportation from the source region to other important regions. The relationship between the ESWs and the MR has become a hot issue recently. The observation of ESWs in association with magnetic reconnection had been widely reported in the magnetotail (Cattell et al. 2005; Deng et al. 2004, 2006; Li et al. 2009, 2012, 2013a, 2013b, 2014a, 2014b) and in the dayside magnetopause (Matsumoto et al. 2003; Deng et al. 2006).

In the process of magnetic reconnection, Cattell et al. (2005) suggested that the electron phase space holes (i.e., electron holes) are excited in the separatrix region, near the outer edge of the current sheet, where there are narrow electron beams, either unidirectional or counterstreaming; Fujimoto and Machida (2006) also suggested that the electron two-stream instability in association

\footnotetext{
* Correspondence: li.shiyou.qiu@gmail.com

College of Aerospace Science and Engineering, National University of Defense Technology, Changsha 410073, China
}

with magnetic reconnection is also responsible for the generation of the electrostatic solitary waves that have been frequently detected in the boundary region. Recently, Lapenta et al. (2011) had conducted massively parallel simulations to study the generation of ESWs in the reconnection process for realistic conditions and for the hydrogen mass ratio in boxes larger than considered in similar previous studies. Their simulation results suggested that along the separatrices, a strong electron flow is observed, sufficient to lead to the onset of streaming instabilities and to form bipolar parallel electric field signatures. In all, to our knowledge, the ESWs can be generated through some kind of mechanism such as the bump-ontail instability or two-stream instability excited by the electron beam which is generated in the MR process.

However, direct evidence of electron beam-excited ESWs in the separatrix is rarely reported in the previous studies. In our previous work, Li et al. (2014a) reported electron beam-associated ESWs in the tailward outflow region near the separatrix of the magnetic reconnection by Geotail observation. The direction of the electron beam on the lobe-side of the separatrix is mainly antiparallel to the ambient magnetic field, and it is mainly parallel on the current sheet-side, and both are consistent with the propagation of ESWs. This suggested a 
good correlation between the propagation direction of the ESWs and the direction of the enhanced high-energy electron flux. However, by single-spacecraft observation, this work cannot distinguish the spatial scale of the electron beam and the ESWs in the separatrix region which is indeed with subtle structure. In the early observation by Cluster, large-amplitude (up to $\sim 50 \mathrm{mV} / \mathrm{m}$ ) ESWs were observed near the outer edge of the plasma sheet during several plasma sheet encounters that have been identified as the passage of a magnetotail reconnection X-line (Cattell et al. 2005). However, the number of ESWs on their study is quite small. Thus, in the present work, we will present the study by multiple spacecrafts of Cluster observation to show the in situ observational evidence of electron beam-associated intense ESWs with large amplitude on the order of $20-30 \mathrm{mV} / \mathrm{m}$ associated with magnetic reconnection in the near-Earth magnetotail. Furthermore, we will make a comparison for the ESWs and electron beams between different satellites.

The organization of this paper is as follows. In the Results and discussion section, we briefly address the magnetic reconnection and separatrix crossing by Cluster firstly and then perform statistical analysis to the ESWs, followed by the study of the electron distribution associated with ESWs, as well as the discussion to the counter direction of the electron beam and ESWs. Conclusions will be presented in the final section.

\section{Methods}

The data onboard the Cluster spacecrafts will be analyzed in this study. Apart from the spin-resolution data from the FluxGate magnetometer (FGM) instrument (Balogh et al. 1997, 2001) and the Cluster Ion Spectrometry (CIS) instrument (Rème et al. 2001), the following two kinds of data are mainly employed: (1) The burst science mode data from the Electric Field and Wave (EFW) instrument (Gustafsson et al. 1997, 2001). The EFW instrument consists of two sets of spherical probe dipoles of length $88 \mathrm{~m}$ in the spin plane of the satellite. There are no electric field measurements along the spin axis. In normal operations, the EFW returns electric field measurements between the opposing probes P1 and P2 (E12) and P3 and P4 (E34) with time resolutions of either $25 \mathrm{~Hz}$ (normal science mode) or $450 \mathrm{~Hz}$ (burst science mode). The EFW data in burst science mode will be used in this study. (2) The pitch angle distribution (PAD) electron data with spin resolution which are obtained from Plasma Electron and Current Experiment (PEACE) (Johnstone et al. 1997). Other kind of data such as the Auroral Electrojet $(\mathrm{AE})$ index is also employed.

In the present work, we will analyze the ESWs observed on the separatrix of the magnetic reconnection event in different satellites of the Cluster tetrahedron. A series of intense ESWs will be continuously observed within $2 \mathrm{~s}$ during the separatrix skimming in the nearEarth magnetotail. A small statistics will be performed for these ESWs on some parameters, i.e., the pulse-topulse amplitude ( $\mathrm{P}-\mathrm{P}$ amplitude), pulse width, pulse interval, and efficiency of symmetry. We will also make comparison for the ESWs and electron beams between different satellites to investigate the relationship between ESWs and electron beam.

\section{Results and discussion}

\section{Event overview}

The magnetic reconnection event is observed by Cluster in the near-Earth magnetotail at about $-15 R_{E}$ on 29 July 2003. This event occurs during a substorm process from 17:10 to 20:00 UT as is seen from the AE index (not shown), which indicates that the substorm evolution process is composed of the growth phase $(\sim 17: 10$ to 18:00 UT), the expansion phase ( 18:10 to 18:50 UT), and the recover phase ( 18:50 to 20:00 UT). Figure 1 overviews this magnetic reconnection event by showing the data obtained from FGM and CIS instruments with a spin resolution, plotting the magnetic components $\left(B_{x}\right.$ and $\left.B_{z}\right)$, the X-component of the plasma velocity $\left(V_{x}\right)$, and the plasma $\beta\left(\beta=2 \mu_{0} \cdot n k T / B_{0}^{2}\right)$ in sequence within the observation time interval of 18:20-18:40 UT. Lines in black, red, green, and blue color in each panel are plotted for spacecraft 1 to 4, respectively (CIS data from SC2 data not available). As seen from the magnetic components, the four satellites detected almost the same phenomenon with a slight difference in the magnetic field and other parameters (will be shown in the following context) which may be caused by the small separation (approximately $\sim 200 \mathrm{~km}$ ) of the Cluster tetrahedron in this event. The Cluster satellites are located in the northern hemispheric side of the current sheet $\left(B_{x}>0\right)$, and with only a small time span, it crosses the neutral current sheet to the southern side at approximately 18:31 UT. The plasma flow $\left(V_{x}\right.$, Fig. 1c) reversal from the large tailward flow $\left(\left|V_{x}\right|_{\max }>500 \mathrm{~km} / \mathrm{s}\right)$ to an intense earthward flow $\left(V_{x, \max }>\approx 500 \mathrm{~km} / \mathrm{s}\right)$ at approximately 18:29 UT within 20 min during this time, as well as the reversal of magnetic field in the Z-components with almost the same tendency of the variation of $V_{x}$, suggests the encountering of a magnetic reconnection region, and the spacecrafts directly cross the reconnection diffusion region from the tailwardside to the earthward-side of it.

The two horizontal lines in Fig. $1 \mathrm{~d}$ denote the value $\beta=$ 0.1 and $\beta=1$, between which is mainly the plasma sheet boundary layer (PSBL) location of spacecraft (Asano et al. 2004). Within an interval of about $10 \mathrm{~min}$ (18:26 to 18:36 UT) before and after the plasma flow reversal, the Cluster stayed only at the boundary layer of the northern hemisphere during most part of the above-mentioned time span since the $\mathrm{X}$-component of magnetic field is 


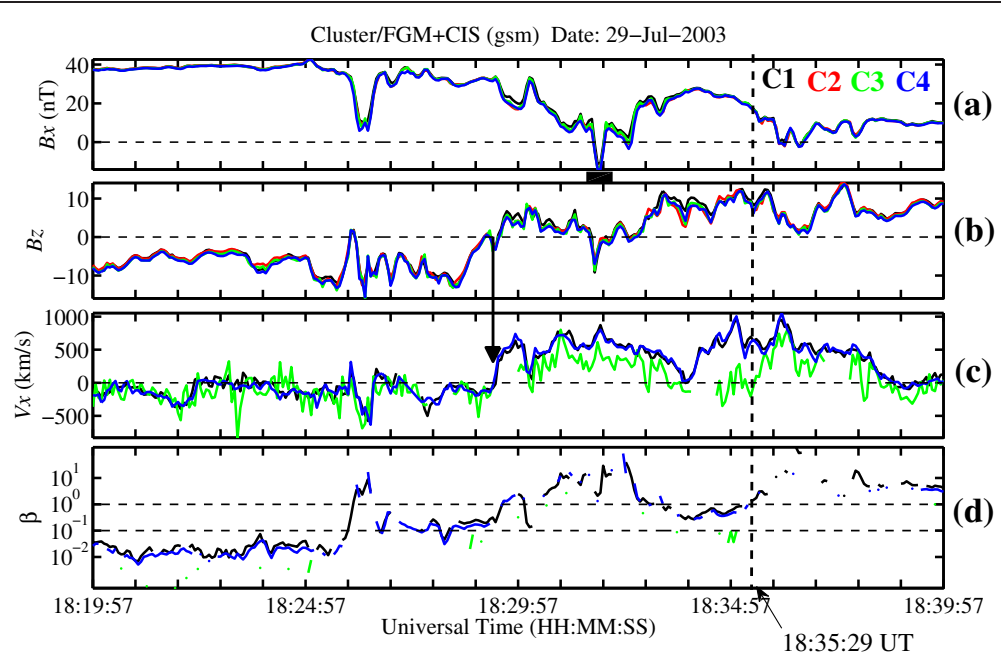

Fig. 1 Overview of the magnetic reconnection event observed by Cluster on 29 July 2003. Top: observation history of Cluster from 18:20 to 18:40 on 29 July 2003. The black, red, green, and blue lines respectively represent the C1, C2, C3, and C4 observations. a-c The X-and Z-components of the magnetic field $\left(B_{x}, B_{z}\right)$ and the X-component of the plasma velocity. The vertical arrow denotes the flow reversal which occurred at $\sim 18: 29$ UT. $\mathbf{d}$ The plasma $\beta$. The two horizontal dotted lines represent $\beta=0.1$ and $\beta=1$, which indicate the plasma sheet boundary layer

comparably large $\left(B_{x} \approx 10-15 \mathrm{nT}\right)$ and the plasma $\beta$ (Fig. 1d) is mainly in the value between 0.1 and 1 (indicated by the two horizontal dashed lines). The orbit of the satellites relative to the diffusion region of the ongoing magnetic reconnection is suggested to be along the separatrix (boundary layer of the reconnection diffusion region) in the tailward-northern-side part and then bypassing the reconnection X-line and finally entering the earthwardnorthern-side.

Recently, Zhou et al. (2014) has also studied this event in the magnetotail mainly focusing on super-Alfvénic electron jet in a reconnection region with a weak guide field. In their study, the northern-southern-northern crossing at around 18:30:43 UT (refer to the pink shaded time interval in Fig. 1 of Zhou et al. (2014) and the horizontal black bar between panels a and b in Fig. 1) was investigated. They presented the in situ evidence of deflected super-Alfvénic electron jet in a reconnection region with a weak guide field, and they also have shown that the electron-scale jet was detected at about 37 ion inertial lengths from the X-line. The corresponding strong electric field in the jet was also studied.

The electron-scale structure is highly sensitive to the guide field. In this event, there is a week guide field, $B_{\text {guide }}=-5.2 \mathrm{nT}$ by averaging the magnetic field $B_{\mathrm{M}}$ during the flow reversal interval between 18:26 and 18:38 UT (Zhou et al. 2014). As will be addressed in the next section, in this study, we only focus on the observation of ESWs within the spin period of 18:35:28 to 18:35:32 UT as indicated by the vertical dashed line in Fig. 1. At this time, the magnitude of plasma $\beta$ for SC1 and SC4 is nearly equal to 1 , indicating that the two spacecrafts are mainly located in the inner side of the PSBL, while the plasma $\beta$ of SC3 is a little less than 0.1 , indicating that SC3 is mainly located in the outer side of the PSBL. In brief, we suggest that at 18:35 UT, the spacecrafts are located on the separatrix of the earthward-northern-side of an ongoing magnetic reconnection diffusion region.

The trajectory of Cluster spacecrafts relative to the magnetic reconnection diffusion region between 18:16 and 18:40 UT is illustrated in Fig. 2. "R1", "R2", "R3," and "R4" are the four subregions of the diffusion region in the $\mathrm{X}-\mathrm{Z}$ plane in the $2-\mathrm{D}$ reconnection model. The dashed cross lines and the solid lines represent respectively the separatrix and the magnetic field lines in the diffusion region. The reconnection jet, out-of-plane magnetic field, and Hall current are respectively denoted by the black thick arrows, the circles with crosses or dots in, and the pink dashed curves in Fig. 2. Note that the trajectory of Cluster spacecraft covers the current sheet twice with a small time interval of 18:31:3018:31:50 UT (refer to the shaded area in Fig. 1 of Zhou et al. (2014)) but cannot be exhibited in this figure. The observations of ESW and the associated electron beam by $\mathrm{C} 2$ on 18:35: 28-32 UT are denoted by the red and purple arrows.

\section{Analysis of ESWs observed by SC2}

By carefully looking through the waveform of the EFW data during the whole reconnection diffusion region crossing process (from 18:20 to 18:40 UT) by the four satellites of Cluster, we find that there are totally approximately 790 cases of electrostatic solitary pulses observed by the four satellites of Cluster during the crossing process 


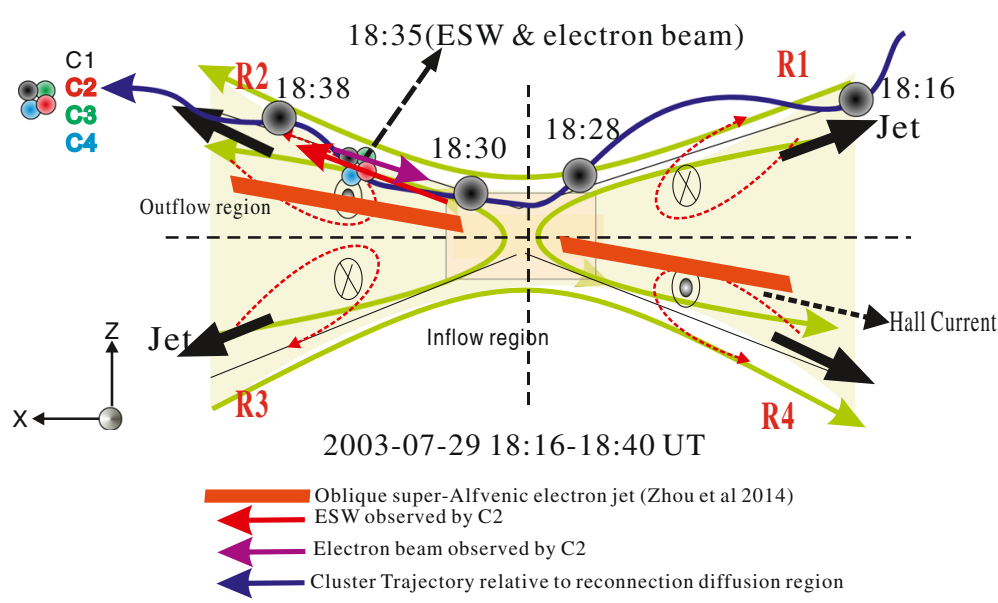

Fig. 2 Illustration of the magnetic reconnection diffusion region within 18:16-18:40 UT interval. The diffusion region is viewed as a traditional ideal 2-D reconnection model where the X-line is the core region of the magnetic reconnection. The observations of ESW and the associated electron beam by C2 on 18:35:28-32 UT are denoted by the red and purple arrows. See the text for detail

to the magnetic reconnection diffusion region within the time scope of the plasma flow burst and reversal. The observation suggests the small-scale electrostatic structure is rather actively associated with the magnetic reconnection in the magnetotail. However, in the present work, we focus only on the observation of ESWs within the spin period of 18:35:28 to 18:35:32 UT because of the special characteristics of ESWs in their spatial structure and the generation mechanism by multi-spacecraft observation which will be carefully addressed in the following context.

We firstly focus on the SC2 observation. Figure 3 plots the parallel component of the electric field $\left(E_{/ /}\right)$of $\mathrm{C} 2$ with the spin period of 18:35:28 to 18:35:32 UT (18:35:28.324 to $18: 35: 31.922$ UT). The data shown in Fig. 3 is the parallel component of the electric waveform derived from the EFW instrument (Gustafsson et al. 1997) by projecting the original observed components $E_{x}$ and $E_{y}$ onto the ambient magnetic field data in the geocentric solar ecliptic (GSE) coordinate which is similar to the SC coordinate. The Z-component of electric field turbulence is assumed to be zero because the ambient field is mainly in the $\mathrm{X}-\mathrm{Y}$ plain of the GSE coordinate. As is seen in the parallel component of the electric field $\left(E_{/ /}\right)$in Fig. 3a, a series of typical isolated bipolar spiky pulses, i.e., ESWs, with the pulse-to-pulse amplitude on the order of $20 \mathrm{mV} / \mathrm{m}$ are observed by SC2 which is located in the separatrix in the earthward-northern-side of the magnetic reconnection X-line.

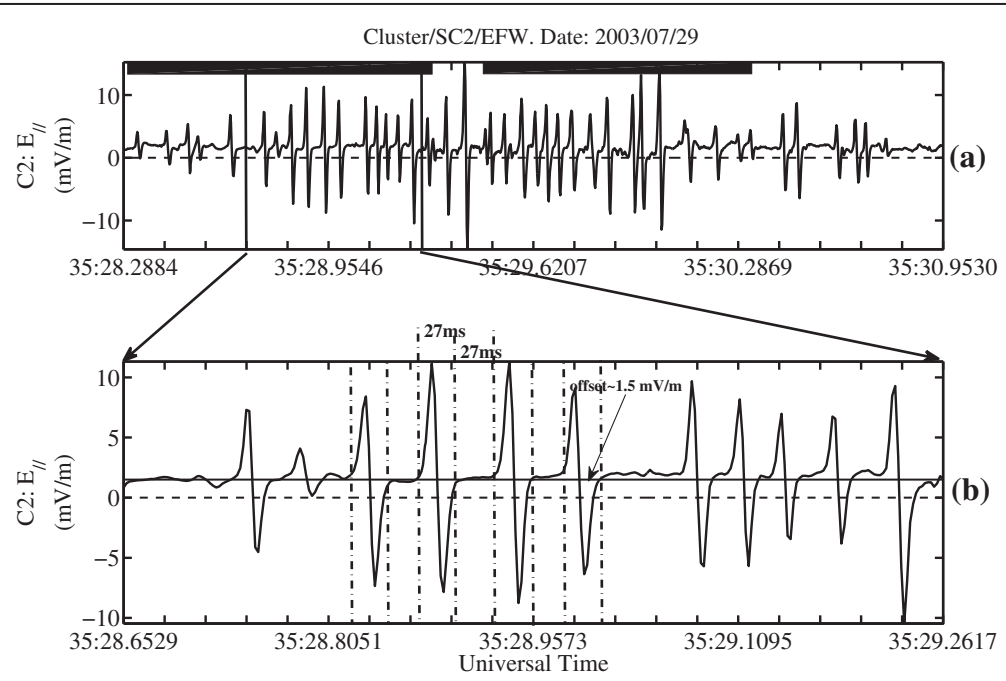

Fig. 3 A series of typical cases of ESWs observed by C2 on the separatrix. a Parallel component of the electric field $\left(E_{/ /}\right)$of $C 2$ with the spin period of 18:35:28 to 18:35:32 UT (18:35:28.324 to 18:35:31.922 UT). b The zoomed-in waveform of $E_{/ /}$between the two solid lines (600 ms from 18:35:28.653 UT) in Fig. 3a. An offset value $(1.5 \mathrm{mV} / \mathrm{m})$ in the amplitude is denoted by the horizontal solid line 
The waveform of $E_{/ /}$between the two solid lines (600 ms from 18:35:28.653 UT) in Fig. 3a is zoomed in in Fig. 3b. The solitary pulses exhibit clear large symmetric structure, with an offset value $(1.5 \mathrm{mV} / \mathrm{m})$ in the amplitude (denoted by the horizontal solid line). There are about 42 pulses of ESWs observed by SC2 (Fig. 3a) within the separatrix location time interval (18:35:28 to 18:35:32 UT) when other spacecrafts are rather nearby. A small statistics is made for these ESWs on some parameters, i.e., the $\mathrm{P}-\mathrm{P}$ amplitude, pulse width, pulse interval, and efficiency of symmetry. The statistical results are displayed in Fig. 4. The four panels respectively show the bar plots for the peak-to-peak amplitude, pulse width, symmetry index, and the pulse interval of ESWs by dividing the corresponding parameters into several value scope. The efficiency of symmetry is interpreted by an index $A_{\text {sym }}$ defined as: $A_{\text {sym }}=\left(E_{\text {max }}-E_{\text {aver }}\right) /\left(E_{\text {aver }}-\right.$ $\left.E_{\text {min }}\right)$,where, $E_{\max }$ and $E_{\min }$ are the values of $E_{/ /}$in the waveform of ESWs at the highest and the flute point, while $E_{\text {aver }}$ is the average value of $E_{/ /}$in the waveform of ESWs. The $A_{\text {sym }}$ represents the efficiency of symmetry of one electron hole on the amplitude. Results show that within these 42 pulses of ESWs on the separatrix, more than half of ESWs are with amplitudes between 10 and $20 \mathrm{mV} / \mathrm{m}$ (Fig. 4a). Most of the ESWs are highly symmetric in waveform with symmetry index between 0.7 and 1.1 (Fig. 4c); The pulse width is as large as 20-45 ms, with most of them between 25 and $30 \mathrm{~ms}$ (Fig. 4b). Most of the ESWs are with pulse interval smaller than $40 \mathrm{~ms}$ (Fig. 4d). These features suggest that the ESWs observed in this case are large intense ESWs with perfect symmetric structure (the efficiency of symmetry is nearly 1) which are different with the previous observation by other probes such as Geotail/WFC in the similar region near/within magnetic reconnection diffusion region (Li et al. 2009).

In the magnetotail, it is usually a positive potential for the ESWs; thus, the polarization in the waveform of ESWs stands for the propagation direction along the ambient magnetic field referring to the spacecraft (Kojima et al. 1999a). However, to infer the propagation direction of the ESW referring to the ambient background magnetic field, the traveling direction of spacecraft should also be taken into consideration. In this case, the Cluster spacecrafts skim the northern boundary layer traveling from the tail-side to the Earth-side relative to the reconnection X-line (refer to Fig. 2); thus, the direction is consistent with the ambient magnetic field. Thus, in this case, the propagation direction of type-A ESWs (negative-to-positive waveform) and type-B ESWs (positive-to-negative waveform) are respectively antiparallel and parallel to the ambient magnetic field (Kojima et al. 1999b; Shin et al. 2008; Li et al. 2014a). In this case, all the waveform of ESWs are of type-B ESWs, i.e., with a propagation direction along the ambient magnetic field. On the boundary layer of the earthward-northernside of the current sheet, the magnetic field is earthward; thus, the ESWs are earthward propagating away from the magnetic reconnection $\mathrm{X}$-line. This is consistent with the previous understanding of ESW outward propagation after generating in the region near the reconnection X-line (Li et al. 2014a).
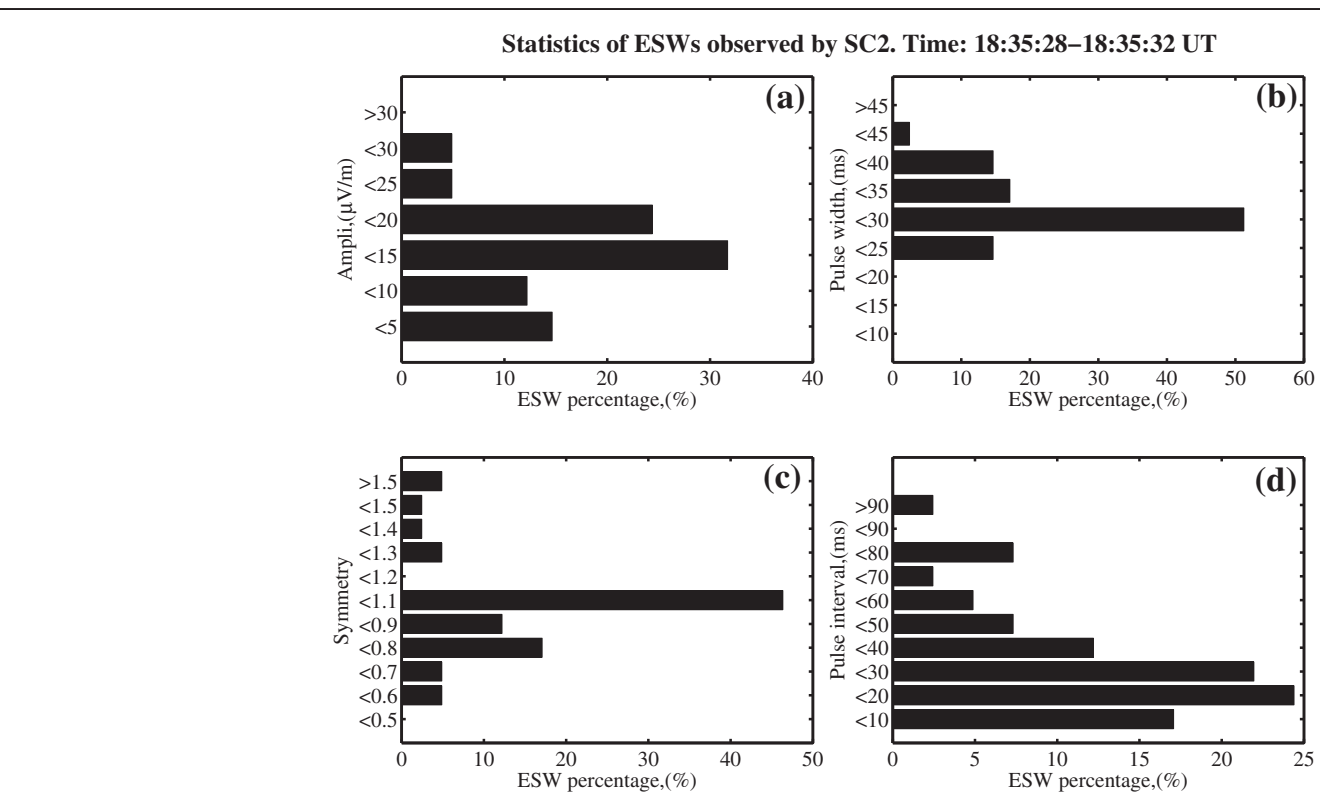

Fig. 4 Statistics of the ESWs observed by SC2 within the separatrix location time interval. a P-P amplitude. b Pulse width. c Efficiency of symmetry. d Pulse interval 
It is noted that the P-P amplitude of the ESWs observed by $\mathrm{C} 2$ in this spin interval exhibits an increasingdecreasing tendency as seen in Fig. 3. In Fig. 3a, the ESWs series can be divided into two series as denoted by the two black bars. In the first series, both the typical pulse width and the pulse interval of ESWs are $27 \mathrm{~ms}$, and the P-P amplitude is as large as $20 \mathrm{mV} / \mathrm{m}$. The increasing-decreasing tendency in the $\mathrm{P}-\mathrm{P}$ amplitude can be clearly seen in Fig. 3b which plots the most part of the first series of ESWs. In this event, the smallamplitude ESWs with small spatial structure observed in the former sequence indicate the electron holes in the early nonlinear evolution process (e.g., Omura et al. 1996; Miyake et al. 1998; Umeda et al. 2002), which indicate that the ESW series with small amplitude may also be closer to the generating site. This conclusion gives evidence that the ESWs propagate toward the Earth along the ambient magnetic field in the Earth-side of the reconnection X-line. The second series of ESWs also exhibit the same tendency but with their parameters slightly smaller than the previous series. We then focus on the spatial scale of the ESWs (i.e., electron holes) observed within this interval of 18:35:28-18:35:31 UT. Forming a tetrahedron with a separation between spacecrafts only about $200 \mathrm{~km}$ in the small separation season, the Cluster spacecrafts present good opportunity for us to study the space structure of the electron phase space hole (electron hole), i.e., the electrostatic solitary wave in the waveform of $E_{/ /}$in the separatrix region, which is with subtle substructure, of magnetic reconnection.

Figure 5 plots the parallel component of the electric field $\left(E_{/ /}\right)$obtained by the EFW instrument on all the four spacecrafts of the Cluster tetrahedron (which is with a small separation between satellites) within the time span of $18: 35: 25.624$ to $18: 35: 31.922$ UT, a time interval covering approximately two spin periods. However, with the second spin period of 18:35:28 to 18:35:32 UT (18:35:28.324 to $18: 35: 31.922$ UT), the ESWs can only be observed by SC2 (refer to Fig. 5b), suggesting that the electron hole must be limited in the tetrahedron of Cluster; thus, the spatial scale in the perpendicular direction should be less than the distance between satellites. During the time interval when SC2 observes ESWs, the distances between SC2 and other satellites are as follows: Dr21 $=270.3 \mathrm{~km}$, Dr23 $=191.1 \mathrm{~km}$, and Dr24 $=238.3 \mathrm{~km}$. Thus, it is clear that the spatial scale of ESWs (i.e., electron holes) must be smaller than $200 \mathrm{~km}$, i.e., $\sim 7.5 d_{e}$, where $d_{e}$ $\approx 26.5 \mathrm{~km}$ is the electron initial scale. It also manifests that the ESWs are mainly excited by electron dynamics.

\section{Electron beam observation and analysis}

Figure 6 plots the sample electron pitch angle distributions for the electron phase space density (PSD) as a function of energy obtained from the pitch angle distribution (PAD) data of the PEACE instrument on board all the four spacecrafts of Cluster within two spin periods from 18:35:25 to 18:35:32 UT. The energy range of PEACE is from $0.59 \mathrm{eV}$ to $26.4 \mathrm{keV}$. The electron distribution data are shown in a magnetic field coordinate system, in which parallel to the local magnetic field is set to $0^{\circ}$ (in black) and $180^{\circ}$ (in green) for the antiparallel direction. The perpendicular direction to the magnetic field is set to $90^{\circ}$ (in red), which is the mean value of pitch angles $75^{\circ} \sim 90^{\circ}$ and $90^{\circ} \sim 105^{\circ}$.

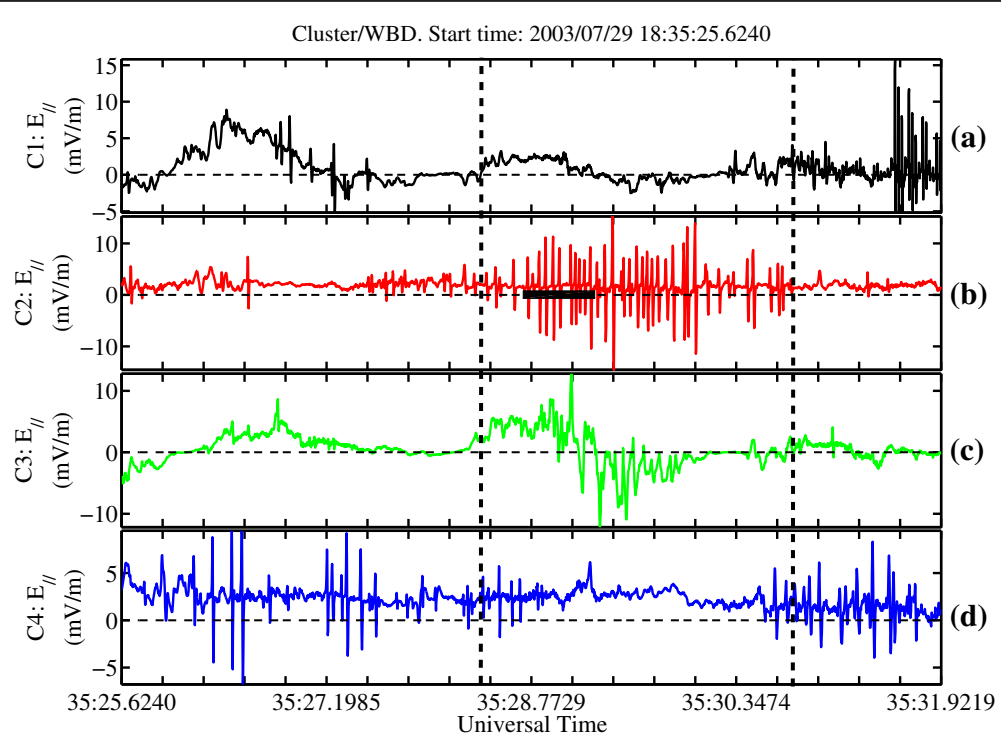

Fig. 5 Overview of the parallel component of the electric field $\left(E_{/ /}\right)$of the four spacecrafts. The $E_{/ /}$is evaluated from the EFW data of four spacecrafts on the separatrix within two spin periods from 18:35:25 to 18:35:32 UT (35:25.624 to 35:31.922 UT). a C1. b C2. c C3. d C4 

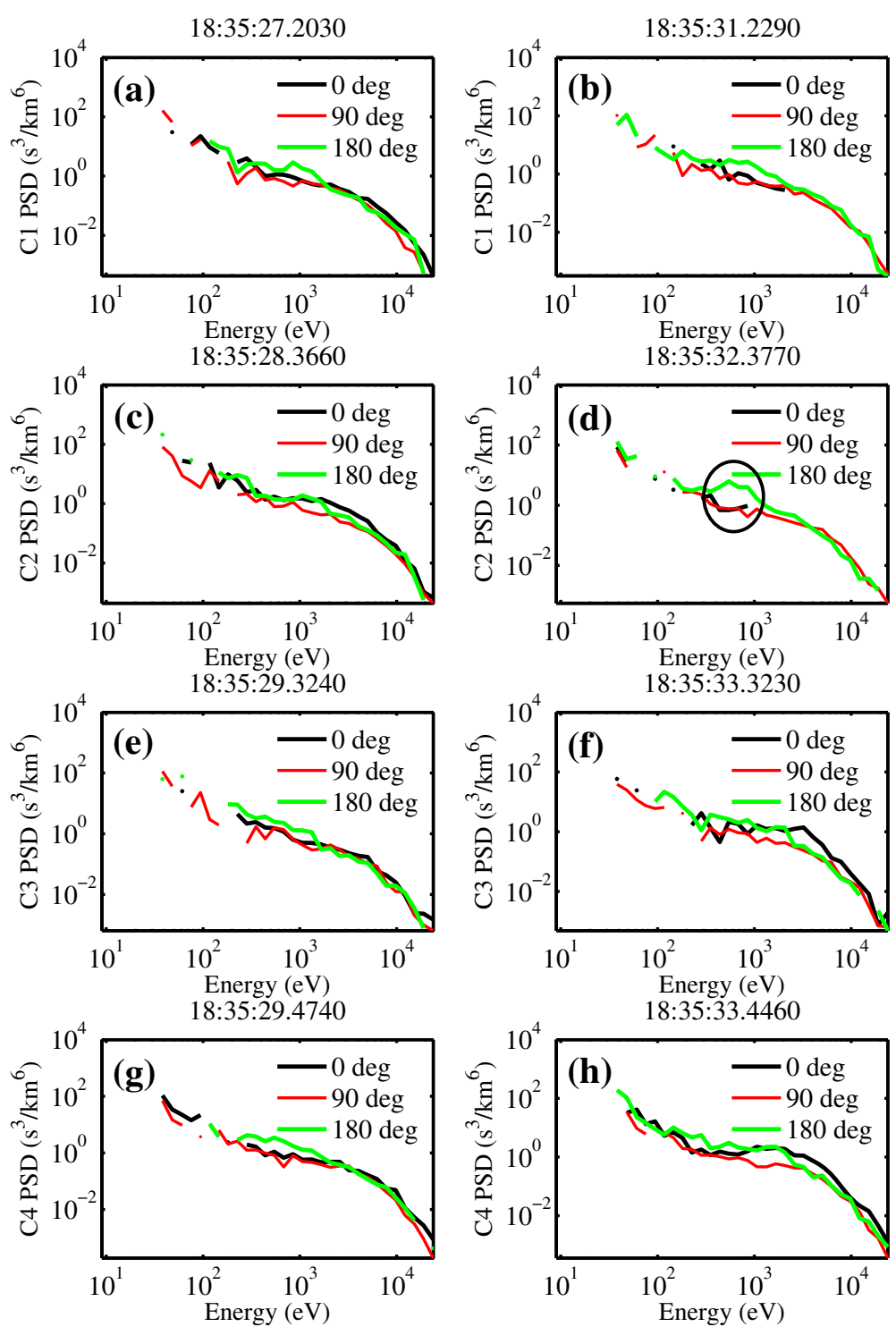

Fig. 6 Distribution of the electron phase space density (PSD) as a function of energy. a, b C1. c, d C2. e, f C3. g, h C4. Panels in the left column show the distribution within the former spin period while the panels in the right column show those in the latter spin period of the four spacecrafts. The black, red, and green lines in each subplot denote the phase space density at $0^{\circ}, 90^{\circ}$, and $180^{\circ}$ pitch angles, respectively

The PSD of the antiparallel velocity distribution (green line) in Fig. $6 \mathrm{~d}$ shows a bump roughly in the range of $0.4-1 \mathrm{keV}$, suggesting that a strong cold electron beam exists and is detected by SC2 during this spin interval. We have also examined the distribution in this spin interval in other directions apart from those in the parallel, antiparallel, and perpendicular directions. However, the electron beam can only be seen in the antiparallel directions. The distribution of this electron beam which overlapped on the background plasma can be fitted by the Maxwellian distribution. Parameters for the beam components are $n_{b}=0.003 \mathrm{~cm}^{-3}, T_{b}=300 \mathrm{eV}, v_{b}=2200$ $\mathrm{km} / \mathrm{s} \approx 0.55 \mathrm{keV}$, where $n_{b}, T_{b}$, and $v_{b}$ are respectively the density, temperature, and the drift speed of the electron beam. The corresponding background plasma is fitted with parameters as follows: $n_{0}=0.04 \mathrm{~cm}^{-3}$ and $T_{0}$ $=3000 \mathrm{eV}$, where $n_{0}$ and $T_{0}$ are respectively the density and temperature of the background plasma. This electron beam is colder than that observed in the density cavity in the magnetic reconnection diffusion region in the presence of a guide field as shown in Zhou et al. (2011), but hotter than the cold counter-streaming electron beams flowing through the hot Maxwellian plasma in the plasma sheet boundary layer measured by Cluster (Teste and Parks 2009) which was also associated with broadband $(\sim 2-6 \mathrm{kHz})$ electrostatic noise.

It is noteworthy that other spacecrafts do not detect such an electron beam even though they are only $190 \sim$ 
$270 \mathrm{~km}$ nearby. This difference indicates that the location of Cluster tetrahedron, i.e., the separatrix region, has a subtle structure within about $200 \mathrm{~km}\left(\sim 7.5 d_{e}\right)$ and the electron beam is limited by the magnetic tube. And of most interest is that only SC2 has observed ESWs within the second spin period of 18:35:28 to 18:35:32 UT. Furthermore, for the SC2 observation, the electron distribution at $4 \mathrm{~s}$ before does not exhibit similar paralleldominated anisotropy distribution. Again, no ESWs are observed by $\mathrm{SC} 2$ during this time interval as shown in Fig. 5b.

Note that the electron phase space distribution function is obtained by integrating the electron amount during a spin period of $4 \mathrm{~s}$. Generally on the observation, it is difficult to address the relationship between the ESWs and the electron beams because the particle measurements do not have the time resolution necessary to identify the electron phase space holes (electron holes) on such short time scales. However, since the observation of ESWs is continuously observed lasting more than $2 \mathrm{~s}$ within this spin period, and cold electron beam is rather strongly observed, we can find the obvious relationship between the two of them. In summary, results from the electron phase space distribution and the EFW observation manifest that the intense ESWs are strongly associated with the cold electron beams of a few hundreds of $\mathrm{eV}(0.4 \sim 1 \mathrm{keV})$ antiparallel to the local magnetic field.

This observational result is similar with the previous understanding of the electron beam near the separatrix identified in observations (Fujimoto et al. 2001; Cattell et al. 2005; Nagai et al. 2011; Li et al. 2014a) and in kinetic simulations (Pritchett 2001; Hoshino et al. 2001). The present observed unidirectional electron beam is a cold beam embedded in the hot background.

\section{Discussion}

ESW is usually regarded to be generated by the twostream electron beam instability or the bump-on-tail instability, which was formed by the high-energy electron beam injecting into the cold electron (Omura et al. 1994, 1996, 1999a; Miyake et al. 1998; Umeda et al. 2002). These instabilities finally lead to the formation of Bernstein-Greene-Kruskal (BGK) (Bernstein et al. 1957) potential structures (i.e., phase space electron holes) which exhibits usually the nonlinear bipolar (sometimes monopolar or tripolar (Li et al. 2013a)) spiky structure in the parallel electric field which was termed as ESWs. In the PSBL region in the magnetotail, the bump-on-tail instability is the most realistic generation mechanism of ESW (Omura et al. 1996). Recent simulation results suggested that the two-stream instability (Che et al. 2009), Buneman instability, and lower hybrid instability (Che et al. 2010) can all generate the ESWs (i.e., the electron holes), but they can be distinguished by the moving speed of the electron holes. During the spin period with ESW observation, the PEACE spinPAD mode data exhibits unidirectional electron beam antiparallel to the ambient field and no electron beam-like distribution is found by other spacecrafts without ESW observation. The present results derived from the electron phase space distribution and the EFW observation manifest that the strong ESWs are closely associated with the cold electron beams of a few hundreds of $\mathrm{eV}(0.4 \sim 1$ $\mathrm{keV}$ ) antiparallel to the local magnetic field. Our study has conquered the restriction of single-spacecraft observation as shown in our previous work by Geotail ( $\mathrm{Li}$ et al. 2014a) and present direct evidence of electron beamassociated ESWs.

Furthermore, since the observation is made when spacecrafts are staying at the northern hemisphere and with earthward plasma flow, the type-B ESWs suggest that the propagation of ESWs is along the ambient magnetic field in the current sheet-side of the separatrix and outwards away from the magnetic reconnection X-line, which is similar with the recent Geotail observation (Li et al. 2014a). We illustrate our observation result in Fig. 2. Cluster/C2 is located on the CS-side of the separatrix in the concerning time interval of 18:35:28-32 UT. It observes outward-traveling ESWs whose direction is denoted by the red arrow, while the simultaneously observed electron beam is antiparallel to the ambient field and is denoted by the purple arrow.

However, it puzzled us that the direction of the electron beam is antiparallel to the ambient magnetic field and is inward toward the reconnection X-line. The generated isolated potentials of ESWs travel along the ambient magnetic field in the same direction of electron beams with the same order of electron beam velocities in computer simulation (Omura et al. 1996; Miyake et al. 1998; Umeda et al. 2002). In our study, the electron beam is counter directed to the traveling direction of ESW. This observation prevents us from interpreting that the ESWs are locally generated by the bump-on-tail instability exhibited by the electron beam.

A candidate interpretation to this case is that the ESWs are not locally generated by the electron beam observed simultaneously. The ESWs can be generated in the region close to the reconnection diffusion region (Drake et al. 2003; Li et al. 2010). By using a particle-incell simulation, Hoshino (2005) discussed that relativistic electrons with $\mathrm{MeV}$ energies are quickly generated in and around the $\mathrm{X}$-type neutral region by utilizing the surfing acceleration, and some electrons can be trapped by the electrostatic potential well of the polarization field inducted by reconnection. As is addressed above, the ESWs possibly originate from the region near the reconnection X-line, which is consistent with the simulation. For the simultaneously observed electron beam in this 
event, since it is antiparallel to the ambient magnetic field, we suggest that the electron beam is not responsible for the generation of the simultaneously observed ESWs. This electron beam is possibly some kind of incoming backstream of electron which is similar to those low-energy electrons flowing toward the reconnection region near the separatrix in previous observation (Fujimoto et al. 2001; Deng et al. 2004) and simulations (e.g., Pritchett 2001; Hoshino et al. 2001).

In observation, Kojima et al. (1999b) performed a statistical study on the propagation direction of ESW and the electron beam on the plasma sheet boundary by Geotail observation. Their results suggested that $88 \%$ of the observed ESW traveling direction is almost the same with the electron beam direction. Their result based on Geotail observation was consistent with the ESW generation mechanism due to the electron beams which resulted from particle simulation performed by Omura et al. (1996) and Miyake et al. (1998). We also note that in the statistics performed by Kojima et al. (1999b), a small portion of the observed ESWs do not travel in the same direction of the electron beam. We suggest that the case in this study corresponds to the small portion of cases in Geotail observation, which only detects that ESWs travel along the magnetic tube with antiparallel electron beam.

As in observation, it is out of the ability to address the counter direction between the ESWs and the electron beam since the two are closely related. It remains open to the next study by computer simulation.

The amplitude of ESWIs represents the potential energy of the electron hole. Though it was reported that large-amplitude (on the order of $100 \mathrm{mV} / \mathrm{m}$ ) ESWs were observed in the magnetopause and bow shock and the cusp region by FAST satellite (Ergun et al. 1998) and by Polar in the low-altitude auroral zone and, at high altitudes $\left(\sim 4-8 \mathrm{R}_{\mathrm{E}}\right)$, during crossings of the plasma sheet boundary and cusp (Cattell et al. 1999, 2003), however, the amplitude of ESWs observed in the magnetotail is usually on the order of $1 \sim 100 \mathrm{uV} / \mathrm{m}$ by Geotail (e.g., Matsumoto et al. 1994, 1999; Kojima et al. 1997, 1999b; Li et al. 2009) and on the order of $0.01 \sim 1 \mathrm{mV} / \mathrm{m}$ by Cluster (Li et al. 2010). Cattell et al. (2005) had observed largeamplitude (up to $50 \mathrm{mV} / \mathrm{m}$ ) solitary waves (electron holes) near the outer edge of the plasma sheet, within and on the edge of a density cavity, and at distances on the order of a few ion inertial lengths from the center of the current sheet. The present cases of ESWIs are strong with large amplitude in the CS-side of the separatrix, consistent with the observation of Cattell et al. (2005) but different with the observation of Geotail in the magnetotail (Li et al. 2009).

The perpendicular scale of the electron holes is not possible to be clearly derived since the perpendicular component is not available; however, since the ESWs are detected only by one spacecraft of the Cluster tetrahedron which separation is smaller than $200 \mathrm{~km}$, the spatial scale in the perpendicular direction should be limited in one small magnetic tube smaller than the max of the separation, i.e., approximately $200 \mathrm{~km}\left(\sim 7.5 d_{e}\right)$. Umeda et al. (2002) had performed a 2-D particle-in-cell (PIC) simulation to the 2-D ESWs and found the tubelike structure of the electron holes. In the observation, Omura et al. (1999b) and Li et al. (2013b) found that many of the PSBL ESWs observed in the near-tall region have large electric fields in perpendicular component relative to the ambient magnetic field. However, we do not have a clear conclusion at this point. The data analysis with EFI on board THEMIS (Angelopoulos 2008) will hurdle this restriction and is currently undertaken.

\section{Conclusions}

In our previous work ( $\mathrm{Li}$ et al. 2014a), strong and weak electron beams are respectively associated with ESWs on the current sheet-side than on the lobe-side of the separatrix based on mono-point observations. In the present work, we have presented an in situ evidence of electron beam-associated ESWs in the separatrix of magnetic reconnection in the near-Earth magnetotail by multispacecraft observation of Cluster. The main conclusions are addressed as follows:

(1)During one spin period, 42 cases of ESWs are continuously observed during $2 \mathrm{~s}$ by SC2 while other spacecrafts do not "detect" them, suggesting that the spatial scale of ESWs is limited in the separation of Cluster (smaller than $200 \mathrm{~km}, \sim 15 d_{e}$ ).

(2) The ESWs exhibit perfect symmetric structure with a small offset about $1.5 \mathrm{mV} / \mathrm{m}$ in the amplitude. A simple statistic is performed to this series of ESWs, and the result reveals that more than half of them are large electron holes with amplitude between 10 and $20 \mathrm{mV} / \mathrm{m}$ and most of the ESWs are highly symmetric in waveform with symmetry index between 0.7 and 1.1 .

(3)During the spin period with ESW observation, the PEACE spinPAD mode data exhibits unidirectional electron beam antiparallel to the ambient field and no electron beam-like distribution is found by other spacecrafts without ESW observation.

(4) The observation with multi-satellite comparison manifests that the ESWs are strongly associated with the cold electron beams of a few hundreds of $\mathrm{eV}$ $(0.4 \sim 1 \mathrm{keV})$ antiparallel to the local magnetic field which is consistent with the bump-on-tail instability.

(5) However, since the electron beam is inward and the ESWs are outward traveling, the relationship between the counter-directed electron beam and the simultaneously observed ESWs remains unclear and open to the next study. 


\section{Abbreviations}

CIS: Cluster Ion Spectrometry; EFW: Electric Field and Wave;

ESWs: Electrostatic solitary waves; FGM: FluxGate magnetometer; PAD: Pitch angle distribution; PEACE: Plasma Electron and Current Experiment; PIC: Particle-in-cell; PSBL: Plasma sheet boundary layer; PSD: Phase space density; MR: Magnetic reconnection.

\section{Competing interests}

This paper presented an in situ evidence of electron beam-associated symmetric bipolar ESWs on the current sheet-side of the separatrix of the magnetic reconnection in the near-Earth magnetotail by multi-spacecraft observation of Cluster. The consistence of the electron beam and ESWs by multiple spacecraft differentiation present clear evidence that the unidirectional electron beam must play a direct and important role on the generation of the ESWs associated with magnetic reconnection.

\section{Authors' contributions}

SL conceived the study and performed most of the data analysis and manuscript composition. SZ and HC helped SL do the analysis and helped in drafting the manuscript. HY helped SL analyze part of the data. All authors read and approved the final manuscript.

\section{Acknowledgements}

The authors appreciate all of the members of the Cluster team for the high quality data and successful operations. We appreciate the Cluster Active Archive (CAA) for providing the data, and we appreciate G. Gustafsson for providing the high resolution EFW data. This work is supported by the National Natural Science Foundation of China under Grant 41304132 and the 53-class General Financial Grant from the China Postdoctoral Science Foundation under Grant 2013M532115.

\section{Received: 14 January 2015 Accepted: 27 May 2015}

Published online: 05 June 2015

\section{References}

Angelopoulos V (2008) The THEMIS mission. Space Sci Rev 141:5-34. doi:10.1007/ s11214-008-9336-1

Asano Y, Mukai T, Hoshino M, Saito Y, Hayakawa H, Nagai T (2004) Current sheet structure around the near-Earth neutral line observed by Geotail. J Geophys Res 109, A02212. doi:10.1029/2003JA010114

Balogh A, Dunlop MW, Cowley SWH, Southwood DJ, Thomlinson JG, The Cluster magnetometer team (1997) The Cluster magnetic field investigation. Space Sci Rev 79:65-91

Balogh A, Carr CM, Acuna MH, Dunlop MW, Beek TJ, Brown P, Fornacon KH, Georgescu E, Glassmeier KH, Harris J, Musmann G, Oddy T, Schwingenschuh K (2001) The Cluster magnetic field investigation: overview of in-flight performance and initial results. Ann Geophys 19:1207-17

Bernstein IB, Greene JM, Kruskal MD (1957) Exact nonlinear plasma oscillations. Phys Rev Lett 108:546-50

Cattell CA, Dombeck J, Wygant JR, Hudson MK, Mozer FS, Temerin MA, Peterson WK, Kletzing CA, Russell CT, Pfaff RF (1999) Comparisons of Polar satellite observations of solitary wave velocities in the plasma sheet boundary and the high altitude cusp to those in the auroral zone. Geophys Res Lett 26:425-8. doi:10.1029/1998GL900304

Cattell C, Neiman C, Dombeck J, Crumley J, Wygant J, Kletzing CA, Peterson WK Mozer FS, Andre' M (2003) Large amplitude solitary waves in and near the Earth's magnetosphere, magnetopause and bow-shock: Polar and Cluster observations. Nonlinear Process Geophys 10:13-26

Cattell C, Dombeck J, Wygant J, Drake JF, Swisdak M, Goldstein ML, Keith W Fazakerley A, André M, Lucek E, Balogh A (2005) Cluster observations of electron holes in association with magnetotail reconnection and comparison to simulations. J Geophys Res 110, A01211. doi:10.1029/2004JA010519

Che H, Drake JF, Swisdak M, Yoon PH (2009) Nonlinear development of streaming instabilities in strongly magnetized plasma. Phys Rev Lett 102(14):145004. doi:10.1103/PhysRevLett.102.145004

Che H, Drake JF, Swisdak M, Yoon PH (2010) Electron holes and heating in the reconnection dissipation region. Geophys Res Lett 37, L11105. doi:10.1029/ 2010GL043608

Deng XH, Matsumoto H, Kojima H, Mukai T, Anderson RR, Baumjohann W, Nakamura R (2004) Geotail encounter with reconnection diffusion region in the Earth's magnetotail: evidence of multiple $X$ lines collisionless reconnection? J Geophys Res 109, A05206. doi:10.1029/2003JA010031

Deng XH, Tang RX, Matsumoto H, Pickett JS, Fazakerley AN, Kojima H, Baumjohann W, Coates A, Nakamura R, Gurnett DA, Liu ZX (2006) Observations of electrostatic solitary waves associated with reconnection by Geotail and Cluster. Adv Space Res 37:1373-81. doi:10.1016/j.asr.2005.05.129

Drake JF, Swissdak M, Cattell CC, Shay MA, Rogers BN, Zeiler A (2003) Formation of electron holes and particle energization during magnetic reconnection. Science 299:873-7. doi:10.1126/science.1080333

Ergun R, Carlson CW, McFadden JP, Mozer FS, Delory GT, Peria W, Chaston C, Temerin M, Elphic R, Strangeway R, Pfaff R, Cattell CA, Klumpa D, Shelley E, Peterson W, Moebius E, Kistler L (1998) FAST satellite observations of largeamplitude solitary wave structures. Geophys Res Lett 25:2041-4

Fujimoto K, Machida S (2006) A generation mechanism of electrostatic waves and subsequent electron heating in the plasma sheet-lobe boundary region during magnetic reconnection. J Geophys Res 111, A09216. doi:10.1029/ 2005JA011542

Fujimoto M, Nagai T, Yokokawa N, Yamade Y, Mukai T, Saito Y, Kokubun S (2001) Tailward electrons at the lobe-plasma sheet interface detected upon dipolarizations. J Geophys Res 106(A10):21255-62. doi:10.1029/2001 JA900011

Gustafsson G, Bostrom R, Holback B, Holmgren G, Lundgren A, Stasiewicz K, Ahlen L, Mozer FS, Pankow D, Harvey P, Berg P, Ulrich R, Pedersen A, Schmidt R, Butler A, Fransen AWC, Klinge D, Thomsen M, Fälthammar CG, Lindqvist PA, Christenson S, Holtet J, Lybekk B, Sten TA, Tanskanen P, Lappalainen K, Wygant J (1997) The electric field and wave experiment for the Cluster mission. Space Sci Rev 79:137-56

Gustafsson G, André M, Carozzi T, Eriksson Al, Fälthammar CG, Grard R, Holmgren G, Holtet JA, Ivchenko N, Karlsson T, Khotyaintsev Y, Klimov S, Laakso H, Lindqvist PA, Lybekk B, Marklund G, Mozer F, Mursula K, Pedersen A, Popielawska B, Savin S, Stasiewicz K, Tanskanen P, Vaivads A, Wahlund JE (2001) First results of electric field and density observations by Cluster EFW based on initial months of operation. Ann Geophys 19:1219-40

Hoshino M (2005) Electron surfing acceleration in magnetic reconnection. J Geophys Res 110, A10215. doi:10.1029/2005JA011229

Hoshino M, Mukai T, Terasawa T, Shinohara I (2001) Suprathermal electron acceleration in magnetic reconnection. J Geophys Res 106(A11):25979-97. doi:10.1029/2001JA900052

Johnstone AD, Alsop C, Burge S, Carter PJ, Coates AJ, Coker AJ, Fazakerley AN Grande M, Gowen RA, Gurgiolo C, Hancock BK, Narheim B, Preece A, Sheather PH, Winningham JD, Woodliffe RD (1997) PEACE: a plasma electron and current experiment. Space Sci Rev 79:351-98

Kojima H, Matsumoto H, Chikuba S, Horiyama S, Ashour-Abdalla M, Anderson RR (1997) Geotail waveform observations of broadband/narrowband electrostatic noise in the distant tail. J Geophys Res 102:14439-55

Kojima H, Matsumoto H, Omura Y (1999a) Electrostatic solitary waves observed in the geomagnetic tail and other regions. Adv Space Res 23:1689-97

Kojima H, Omura Y, Matsumoto H, Miyaguti K, Mukai T (1999b) Characteristics of electrostatic solitary waves observed in the plasma sheet boundary: statistical analysis. Nonlin Processes Geophys 6:179-86

Lapenta G, Markidis S, Divin A, Goldman MV, Newman DL (2011) Bipolar electric field signatures of reconnection separatrices for a hydrogen plasma at realistic guide fields. Geophys Res Lett 38, L17104. doi:10.1029/2011GL048572

Li SY, Deng XH, Zhou M, Tang RX, Liu K, Kojima H, Matsumoto H (2009) Statistical study of electrostatic solitary waves associated with reconnection: Geotail observations. J Adv Space Res 43(3):394-400. doi:10.1016/j.asr.2008.05.013

Li SY, Deng XH, Zhou M, Yuan ZG, Wang JF, Lin X, Lin MH, Fu S (2010) Cluster observation of electrostatic solitary waves around magnetic null point in thin current sheet. Chin Phys Lett 27(1):019401

Li SY, Zhang SF, Deng XH, Cai H (2012) Spatial evolution of electrostatic solitary waves along plasma sheet boundary layer adjacent to magnetic reconnection X-line. Chin Phys Lett 29(8):089402

Li SY, Zhang SF, Cai H, Chen XQ, Deng XH (2013a) Statistics of the tri-polar electrostatic solitary waves within magnetic reconnection diffusion region in the near-Earth magnetotail. J Geophys Res 118:2911-8. doi:10.1002/jgra.50209

Li SY, Zhang SF, Deng XH, Cai H (2013b) Large bi-polar signature in a perpendicular electric field of two-dimensional electrostatic solitary waves associated with magnetic reconnection: statistics and discussion. Chin Phys Lett 30(1):019401

Li SY, Omura Y, Lembège B, Deng XH, Kojima H, Saito Y, Zhang SF (2014a) Geotail observation of counter directed ESWs associated with the separatrix of magnetic reconnection in the near-Earth magnetotail. J Geophys Res Space Physics 119:202-10. doi:10.1002/2013JA018920 
Li SY, Zhang SF, Cai H, Yu SF (2014b) Concentration of electrostatic solitary waves around magnetic nulls within magnetic reconnection diffusion region: single-event-based statistics. Earth Planets and Space 66:161. doi:10.1186/s40623-014-0161-3

Matsumoto H, Kojima H, Miyatake T, Omura Y, Okada M, Nagano I, Tsutsui M (1994) Electrostatic solitary waves (ESW) in the magnetotail: BEN waveforms observed by Geotail. Geophys Res Lett 21:2915

Matsumoto H, Frank LA, Omura Y, Kojima H, Paterson WR, Tsutsui M, Anderson RR, Horiyama S, Kokubun S, Yamamoto T (1999) Generation mechanism of ESW based on GEOTEIL plasma wave observation, plasma observation and particle simulation. Geophys Res Lett 26(3):421-4

Matsumoto H, Deng XH, Kojima H, Anderson RR (2003) Observation of electrostatic solitary waves associated with reconnection on the dayside magnetopause boundary. Geophys Res Lett 30:1326. doi:10.1029/2002GL016319

Miyake T, Omura Y, Matsumoto H, Kojima H (1998) Two-dimensional computer simulations of electrostatic solitary waves observed by Geotail spacecraft. J Geophys Res 103(A6):11841-50. doi:10.1029/98JA00760

Nagai T, Shinohara I, Fujimoto M, Matsuoka A, Saito Y, Mukai T (2011) Construction of magnetic reconnection in the near-Earth magnetotail with Geotail. J Geophys Res 116, A04222. doi:10.1029/2010JA016283

Omura Y, Kojima H, Matsumoto H (1994) Computer simulation of electrostatic solitary waves: a nonlinear model of broadband electrostatic noise. Geophys Res Lett 21(25):2923-6

Omura Y, Matsumoto H, Miyake T, Kojima H (1996) Electron beam instabilities as generation mechanism of electrostatic solitary waves in the magnetotail. J Geophys Res 101(A2):2685-97. doi:10.1029/95JA03145

Omura Y, Kojima H, Miki N, Mukai T, Matsumoto H, Anderson R (1999a) Electrostatic solitary waves carried by diffused electron beams observed by Geotail spacecraft. J Geophys Res 104(A7):14627-37. doi:10.1029/1999JA900103

Omura Y, Kojima H, Miki N, Matsumoto H (1999b) Two-dimensional electrostatic solitary waves observed by Geotail in the magnetotail. Adv Space Res 24(1):55-8

Pritchett PL (2001) Geospace Environment Modeling magnetic reconnection challenge: simulations with a full particle electromagnetic code. J Geophys Res 106(A3):3783-98. doi:10.1029/1999JA001006

Rème H, Aoustin C, Bosqued JM, Dandouras I, Lavraud B, Sauvaud JA et al (2001) First multispacecraft ion measurements in and near the Earth's magnetosphere with the identical Cluster ion spectrometry (CIS) experiment. Ann Geophys 19:1303

Shin K, Kojima H, Matsumoto H, Mukai T (2008) Characteristics of electrostatic solitary waves in the Earth's foreshock region: Geotail observations. J Geophys Res 113, A03101. doi:10.1029/2007JA012344

Teste A, Parks GK (2009) Counter-streaming beams and flat-top electron distributions observed with Langmuir, Whistler, and compressional Alfvén waves in Earth's magnetic tail. Phys Rev Lett 102:075003. doi:10.1103/PhysRevLett.102.075003

Umeda T, Omura Y, Matsumoto H, Usui H (2002) Formation of electrostatic solitary waves in space plasmas: particle simulations with open boundary conditions. J Geophys Res 107:1449. doi:10.1029/2001JA000286

Zhou M, Pang Y, Deng XH, Yuan ZG, Huang SY (2011) Density cavity in magnetic reconnection diffusion region in the presence of guide field. J Geophys Res 116, A06222. doi:10.1029/2010JA016324

Zhou M, Deng X, Tang R, Pang Y, Xu X, Yuan Z, Huang S (2014) Evidence of deflected super-Alfvénic electron jet in a reconnection region with weak guide field. J Geophys Res 119:1541-8. doi:10.1002/2013JA019556

\section{Submit your manuscript to a SpringerOpen ${ }^{\circ}$ journal and benefit from:}

- Convenient online submission

- Rigorous peer review

- Immediate publication on acceptance

- Open access: articles freely available online

- High visibility within the field

- Retaining the copyright to your article

Submit your next manuscript at $>$ springeropen.com 